Vol. 11 (29), pp. 1171-1183, 7 August, 2017

DOI: $10.5897 / A J M R 2016.8256$

Article Number: 6648E3F65431

ISSN 1996-0808

Copyright (C) 2017

African Journal of Microbiology Research

Author(s) retain the copyright of this article

http://www.academicjournals.org/AJMR

\title{
Midgut bacterial diversity analysis of laboratory reared and wild Anopheles gambiae and Culex quinquefasciatus mosquitoes in Kenya
}

\author{
Erastus Mwaringa Mwadondo ${ }^{1}$, Amanuel Ghilamicael ${ }^{2}$, Amos Ematiti Alakonya ${ }^{2,3}$ and \\ Remmy Wekesa Kasili ${ }^{2 *}$
}

\footnotetext{
${ }^{1}$ Department of Zoology, Jomo Kenyatta University of Agriculture and Technology, P. O. Box 62000-00200, Nairobi, Kenya.

${ }^{2}$ Institute for Biotechnology Research, Jomo Kenyatta University of Agriculture and Technology P. O. Box 62000-00200, Nairobi, Kenya.

${ }^{3}$ International Institute of Tropical Agriculture, Headquarters, PMB, 5320, Ibadan Oyo State, Nigeria.
}

Received 5 August, 2016; Accepted 21 June, 2017

\begin{abstract}
Midgut symbiotic bacteria are known to play fundamental roles in the biology of mosquitoes, however knowledge of midgut bacterial communities associated with mosquitoes is scanty due to limitation of the isolation techniques based on culturing. In this study, the composition and diversity of midgut bacteria in field collected and lab reared adult female Anopheles gambiae and Culex quinquefasciatus mosquitoes was explored using the Illumina sequencing. Deoxyribonucleic acid was isolated from the pooled midgut extracts and their 16S rRNA gene sequenced using Illumina sequencing platform. Operational taxonomic units (OTUs) were analyzed using QIIME 1.8.0; taxonomy was assigned using BLASTn against SILVA 119 and hierarchical clustering was done using R program software. Out of the total number of sequence reads obtained, 145 OTUs were realized at $3 \%$ genetic distance. The 145 OTUs spanned 12 phyla; Proteobacteria, Firmicutes, Cyanobacteria, Euryarchaeota, Gemmatimonadetes, Spirochaetae, Archeabacteria Verrucomicrobia, Chloroflexi, Bacteriodetes, Acidobacteria and Actinobacteria. Microbial community composition based on OTUs showed significant difference between field collected and lab reared mosquitoes $\left(x^{2}=45.0799, p=3.2 \times 10^{-5}\right)$. Similarly, there was a significant difference in community composition at OTU level between Anopheles gambiae and Culex quinquefasciatus $\left(x^{2}=31.2257, p=7.7 \times 10^{-4}\right)$. The bacterial composition and diversity appeared to be influenced by the environment and the species of the mosquitoes.
\end{abstract}

Key words: Anopheles gambiae, Culex quinquefasciatus, midgut, DNA, diversity.

\section{INTRODUCTION}

Mosquitoes transmit diseases like malaria, dengue, lymphatic filariasis, yellow fever among others. Among these diseases, malaria is the most important mosquito borne disease with an estimated 214 million new cases of malaria worldwide (World Health Organization (WHO),
2015). The African region accounted for most of the global cases of malaria (88\%), followed by South-East Asia region (10\%) and Eastern Mediterranean region (2\%) (WHO, 2015). In Kenya, there were an estimated 6.7 million new clinical cases and 4,000 deaths each year 
and those living in Western Kenya have an especially high risk of malaria (Centers for Disease Control and Prevention (CDC), 2015). Most of the deaths are caused by the parasite Plasmodium falciparum whose major vector in Africa is the mosquito species Anopheles gambiae that is widely distributed throughout the Afrotropical belt (Boissière et al., 2012).

Another mosquito species Culex quinquefasciatus is the principal vector for Wuchereria bancrofti, the filarial worm that causes filariasis and Japanese encephalitis (Agrawal and Sashindran, 2006). Lymphatic filariasis is a major public health problem worldwide. It is estimated that 1.3 billion people from 83 countries are living with the disease or are at risk of infection (Agrawal and Sashindran, 2006). Lymphatic filariasis is present on the East African coast especially in Kenya (Njenga et al., 2011).

Current mosquito vector control strategies include insecticide treatment delivered through spraying houses and insecticide-impregnated mosquito nets. While these methods are effective at decreasing mosquito vector numbers, they have also contributed to the rise in insecticide resistant mosquitoes (Bando et al., 2013).

Various alternative approaches are being tried to reduce malaria cases in the world, and one such approach is paratransgenesis. Paratransgenesis is a method where by a symbiotic bacteria is used to express effector molecules inside a targeted vector. The symbiotic bacteria are genetically modified to produce effector molecules and then reintroduced into the mosquito to produce the required effect (Chavshin et al., 2012). Understanding the microbial community structure of the mosquito midgut is therefore necessary in order to identify possible bacterial candidates for paratransgenesis. The mosquito midgut plays a critical role to the survival and development of the parasites and is therefore, the most attractive site to target malaria parasites (Whitten et al., 2006). The midgut microbiota of mosquitoes is still not well investigated and a few studies have been carried out on microflora of wild caught malaria vectors (Wang et al., 2011). The available conventional culture techniques limit the scope in determination of the microbial diversity since it sometimes misses out on non-culturable microbes (Pidiyar et al., 2004).

In laboratory-raised mosquitoes, the midgut bacteria can be acquired through contaminated sugar solutions, blood meals and transmitted transstadially. However, in wild mosquitoes, the origin of the midgut bacteria, is still unknown (Riehle and Jacobs-Lorena, 2005). In the current study the bacterial composition and diversity in the midgut of lab reared and field collected $A$. gambiae and $C$. quinquefasciatus mosquitoes were determined using the illumina sequencing.

\section{MATERIALS AND METHODS}

\section{Study site}

The study area for field collected mosquitoes was Ahero, Kenya, which is a malaria endemic region. It is located at latitude $0^{\circ} 11^{\prime} \mathrm{S}$ and longitude $34^{\circ} 55^{\prime} \mathrm{E}$ and is approximately $1153 \mathrm{~m}$ above sea level. The area has a tropical climate with significant rainfall throughout the year and with an average temperature of $23.0^{\circ} \mathrm{C}$.

\section{Collection of field $A$. gambiae and C. quinquefasciatus mosquitoes}

Adult $A$. gambiae and $C$. quinquefasciatus mosquitoes were captured from pit shelters by use of Centre for Disease Control and Prevention (CDC) light traps. The CDC light traps were hung at least one meter above the ground on a tree or pole between 6:00 and 7:00 pm in the evening and left overnight. The collection bags containing the mosquitoes were picked between 6.00 and 6.30 am in the morning. The mosquitoes were then put into vial/jars from the collection bags using mouth aspirators and stored at $4^{\circ} \mathrm{C}$. One hundred and thirty eight adult female Anopheles gambiae and Culex quinquefasciatus mosquitoes were identified to species level using a standard morphological key according to Gillies and De Meillon, (1968). The specimens were then transferred to the laboratory at the Institute for Biotechnology Research, Jomo Kenyatta University of Agriculture and Technology (JKUAT).

\section{Acquisition of laboratory-reared $A$. gambiae and $C$. quinquefasciatus}

One hundred and thirty eight laboratory reared adult female $A$. gambiae and $C$. quinquefasciatus mosquitoes were acquired from the International Centre of Insect Physiology and Ecology (ICIPE) Kasarani, Nairobi. They were transferred live to the laboratory at the Institute for Biotechnology Research, (JKUAT) and maintained in a mosquitarium at $28^{\circ} \mathrm{C}$ and 70 to $80 \%$ humidity until dissection. The mosquitoes were offered resins and $1 \%$ glucose solution as a source of energy and were not fed on blood.

\section{Dissection of mosquitoes and isolation of DNA}

Dissection of mosquitoes was done according to Rani et al. (2009). Before dissecting, the mosquitoes were chilled to death and sterilized with $70 \%$ ethanol then transferred into sterile distilled water in a sterile hood. The mosquitoes were dissected individually under sterile conditions. The midguts were mashed and suspended in $100 \mu \mathrm{l}$ of sterile phosphate buffered solution (PBS). The mashed midguts were ground to homogeneity. Each midgut extract consisted of 20 pooled midguts of adult female mosquitoes. Field collected and lab reared mosquitoes had seven pooled midgut extracts each. The midgut extracts were stored at $80^{\circ} \mathrm{C}$ until further analysis.

The total microbial genomic DNA was extracted separately from each group of mosquito midgut extracts using purelink genomic DNA mini kit (Invitrogen), following the manufacturer's

\section{*Corresponding author: E-mail: rkasili@gmail.com. Tel: +254713800972.}


instructions (CAT number, K1820-02 Life technologies, California, USA). Genomic DNA concentration was quantified using a nano drop spectrophotometer and the DNA stored at $-20^{\circ} \mathrm{C}$ until further analysis.

\section{Polymerase chain reaction amplification}

Polymerase chain reaction (PCR) amplification of the 16S rRNA gene V4 variable region was carried out on the extracted DNA using primers 515F (GTGCCAGCMGCCGCGGTAA) and 806R (GGACTACHVGGGTWTCTAAT) that had a barcode (Caporaso et al., 2010). PCR amplification was carried out in 30 cycles using the HotStarTaq Plus Master Mix Kit (Qiagen, USA) under the following conditions: $94^{\circ} \mathrm{C}$ for $3 \mathrm{~min}$ of intitial heating, followed by 30 cycles of $94^{\circ} \mathrm{C}$ for $30 \mathrm{~s}, 53^{\circ} \mathrm{C}$ for $40 \mathrm{~s}$ and $72^{\circ} \mathrm{C}$ for $1 \mathrm{~min}$, followed by a final elongation step at $72^{\circ} \mathrm{C}$ for $5 \mathrm{~min}$. PCR products were visualized on $2 \%$ agarose gel to determine the success of amplification and the relative intensity of bands. Multiple samples were pooled together in equal proportions based on their DNA concentrations from the gel images. Pooled samples were purified using calibrated Ampure XP beads (Agencort Bioscience Corporation, MA, USA).

\section{Amplicon library preparation}

The pooled and purified PCR products were used to prepare DNA library by following Illumina TruSeq DNA library protocol (Yu and Zhang, 2012). Sequencing was performed at Molecular Research DNA (www.mrdnalab.com, Shallowater, TX, USA) on a MiSeq platform following the manufacturer's guidelines. The resulting raw sequences were submitted to NCBI (Sequence Read Archive) with the following study accession numbers; sequences for field collected $A$. gambiae SAMN04386463; field collected $C$. quinquefasciatus SAMN04386464; lab reared $A$. gambiae SAMN04386465 and lab reared $C$. quinquefasciatus SAMN04386466.

\section{Sequence analysis and taxonomic classification}

Sequences obtained from the Illumina sequencing platform were depleted of barcodes and primers using a proprietary pipeline (www.mrdnalab.com, MR DNA, Shallowater, TX) developed at the service provider's laboratory. Short sequences $<200 \mathrm{bp}$, sequences with ambiguous base calls, and those with homopolymer runs exceeding 6 bp were removed. The sequences were denoised, chimeras and singleton sequences removed (Capone et al., 2011; Dowd et al., 2011; Eren et al., 2011). De novo OTU clustering was done with standard UCLUST method using the default settings as implemented in QIIME Version 1.8.0 at $97 \%$ similarity level (Caporaso et al., 2010). Taxonomy was assigned to each OTU using BLASTn against SILVA SSU Reference 119 database at default e-value threshold of 0.001 in QIIME (Quast et al., 2013).

\section{Diversity indices}

Diversity indices (Shannon, Inverse Simpson, Evenness), rarefaction, Venn diagram (to compare the shared OTUs between the samples of mosquitoes) and hierarchical clustering were computed, using Vegan package version 1.16 to 32 in $\mathrm{R}$ software ( $\mathrm{R}$ development Core Team, (2012). Kruskal-Wallis rank sum test was used to compare the relative abundance of gut microflora among $A$. gambiae and $C$. quinquefasciatus from lab reared and field collected samples using $R$ programming language ( $R$ development Core Team (2012). Significance was tested at 95\% confidence interval $(p=0.05$ ). To support OTU-based analysis, taxonomic groups were derived from the number of reads assigned to each taxon at all ranks from domain to species using the taxa_summary.txt output from QIIME pipeline Version 1.8.0.

\section{RESULTS}

\section{Assemblage and diversity of the microbial communities}

After removing chimeras, denoising and demutiplexing, a total of 24,025 sequence reads greater than 200 bp were attained from the $16 \mathrm{~S}$ rRNA data. Total OTU richness at $3 \%$ distance amounted to 145 . The OTUs per data set ranged between 26 and 102. OTUs comprised $87 \%$ bacteria, $0.7 \%$ Archaea, 2\% Fungi, 1.4\% Eukarya and $8 \%$ no blast hit (sequences reads that were not assigned). Rarefaction curve was plotted in order to evaluate if all the diversity within the samples had been exhaustively recovered (Figure 1).

The slopes of the curves flatten out in cases where full diversity has been detected. This indicates that even if more sequences were obtained, the number of OTUs detected in the samples would not increase. However, more sequences would be required to exhaust the full diversity within the samples if the slopes did not flatten out (Chao et al., 2014). The sequencing depth as shown by the rarefaction curve was exhaustive enough to ensure the inclusion of the entire diversity of the microbes in the midgut of the two species of mosquitoes collected from field and lab reared.

The distribution of shared OTUs across the two species of mosquitoes and the sample source (lab reared and field collected) is shown in (Figure 2). Seven OTUs were common in all the samples, fifty four (54) OTUs were only found in field collected $A$. gambiae while 18 OTUs were detected only from the field collected $C$. quinquefasciatus samples. Lab reared $C$. quinquefasciatus and $A$. gambiae samples had one and 10 unshared OTUs, respectively.

A diversity index is a quantitative measure that reflects how many different types of species there are in a community and simultaneously takes into account how evenly the individuals are distributed among them. The estimated Shannon diversity index varied between (3.54) for field collected A. gambiae and (1.93) for lab reared $C$. quinquefasciatus (Table 1). The Shannon diversity index for field collected $C$. quinquefasciatus (2.73) was higher than lab reared $A$. gambiae (2.52) and lab reared $C$. quinquefasciatus (1.93). The Shannon index is a representation of species abundance and evenness, when either of these two factors increases, the diversity index also increases. Evenness index was used to estimate how well the species are evenly distributed in a community. The highest evenness was recorded from field collected $A$. gambiae (0.767) indicating that OTUs were evenly distributed as compared to other samples. The lowest evenness was recorded from lab reared $C$. 


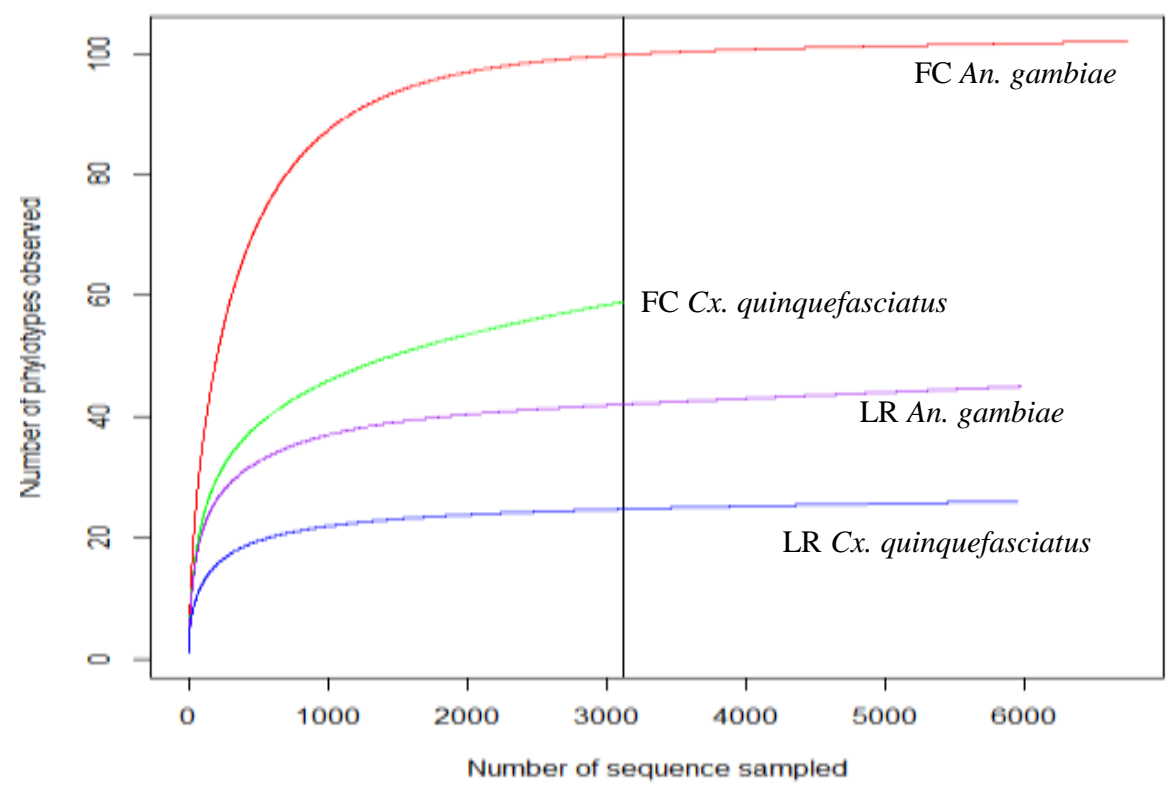

Figure 1. Rarefaction curve analysis in field collected (FC) $C x$. (Culex) and lab reared (LR) An. (Anopheles) samples.

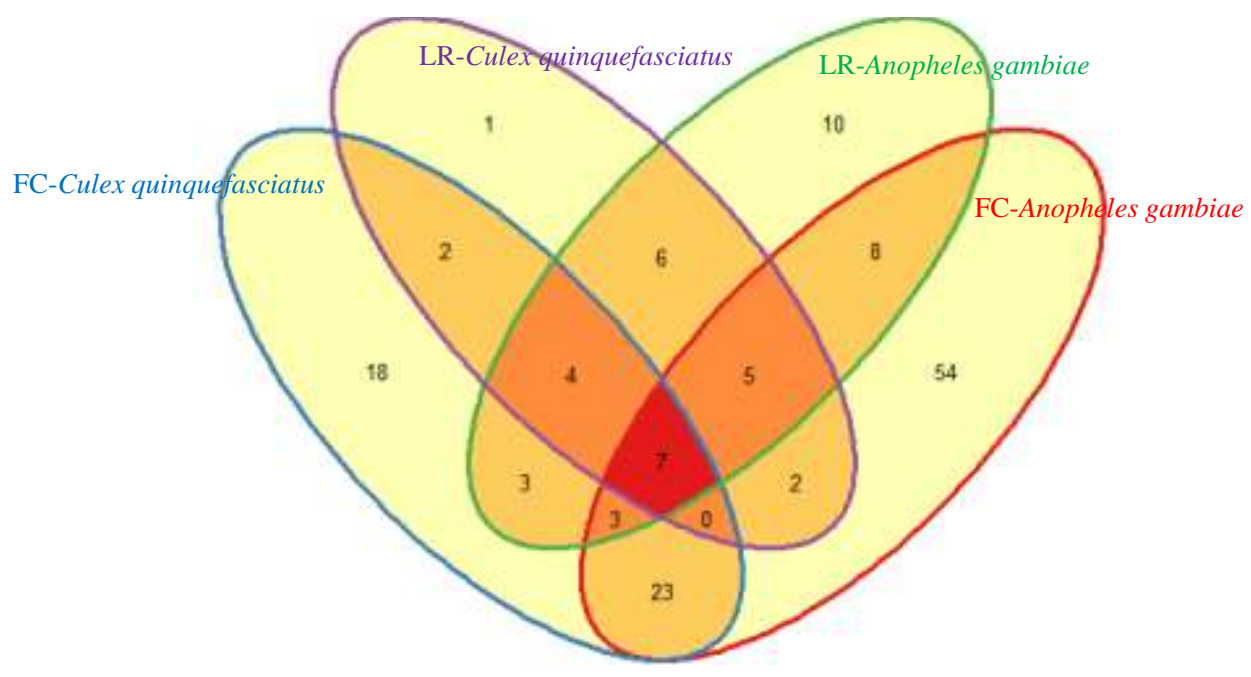

Figure 2. Venn diagram showing the distribution of shared OTUs across the 4 samples. Numbers indicate OTUs enumerated in field collected (FC) and lab reared (LR) samples.

Table 1. Diversity indices computed at OTU-based bacterial taxonomic units obtained from samples of the Field collected (FC) and Lab reared $(\mathrm{LR})$ mosquitoes.

\begin{tabular}{lcccccc}
\hline Sample & $\begin{array}{c}\text { No. of sequences } \\
\text { after filtering }\end{array}$ & $\begin{array}{c}\text { Richness } \\
\text { (OTUs) }\end{array}$ & $\begin{array}{c}\text { Shannon } \\
\text { (H) }\end{array}$ & $\begin{array}{c}\text { Inverse } \\
\text { Simpson }\end{array}$ & $\begin{array}{c}\text { Evenness } \\
\text { (J) }\end{array}$ & $\begin{array}{c}\text { Effective no. } \\
\text { of sp. }\end{array}$ \\
\hline FC Anopheles gambiae & 7516 & 102 & 3.54 & 19.98 & 0.767 & 34.47 \\
FC Culex quinquefasciatus & 3465 & 59 & 2.73 & 8.72 & 0.67 & 15.33 \\
LR Anopheles gambiae & 6669 & 45 & 2.52 & 5.98 & 0.661 & 12.43 \\
LR Culex quinquefasciatus & 6375 & 26 & 1.93 & 4.65 & 0.593 & 6.89 \\
Total & $\mathbf{2 4 , 5 0 5}$ & $\mathbf{1 4 5}$ & & & & \\
\hline
\end{tabular}

The microbial community composition, based on Kruskal-Wallis test, at OTU level showed significant difference between field collected and lab reared mosquitoes $\left(x^{2}=45.0799, p=3.2 \times 10^{-5}\right)$. Similarly, there was significant difference in microbial community composition at OTU level between Anopheles gambiae and Culex quinquefasciatus $\left(x^{2}=31.2257, p=7.7 \times 10^{-4}\right)$. 


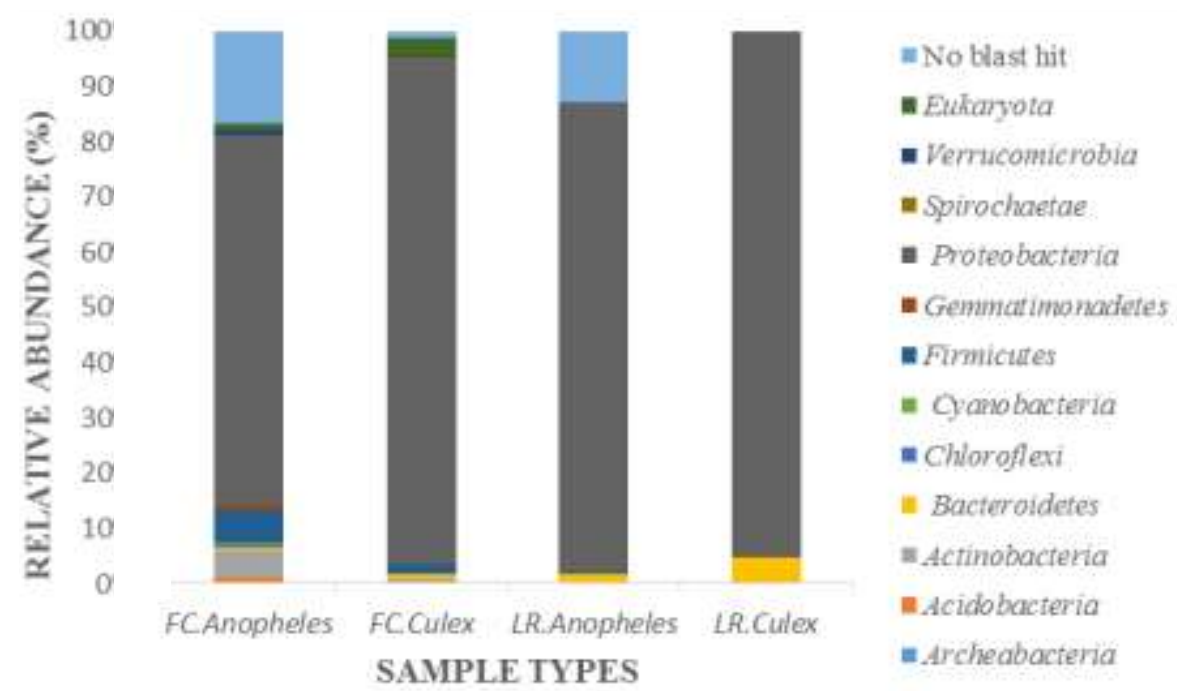

Figure 3. Relative abundance at phylum level from the field collected (FC) and lab reared $(\mathrm{LR})$ samples.

quinquefasciatus (0.593) indicating that bacterial species are less evenly distributed and some species are more dominant than the others. The value of Inverse Simpson index ranged from 4.65 for lab reared $C$. quinquefasciatus to 19.98 for field collected $A$. gambiae. The value of Inverse Simpson index was observed to increase with increase in diversity.

\section{Microbial taxonomic community composition}

The SILVA SSU Reference 119 database (Quast et al., 2013) was used to assign reads to appropriate taxonomic ranks. The OTUs spanned 12 phyla (Figure 3); Proteobacteria (62.04-95.11\%), Firmicutes (0.00-6.13 $\%)$, Bacteriodetes (0.42-4.89\%), Actinobacteria (0.00$4.97 \%)$, Eukaryota (0.00-3.46\%), Gemmatimonadetes (0.00-0.86\%), Spirochaetae (0.00-0.21\%), Verrucomicrobia (0.00-0.76\%), Chloroflexi $(0.00-0.80 \%)$, Acidobacteria (0.00-0.68\%), Archeabacteria (0.00-0.39\%) and Cyanobacteria (0.00-0.10\%). The no blast hits had relative abundance ranging from 0.00 to $16.58 \%$.

OTUs belonging to the Phylum Proteobacteria were the most abundant and were represented by the most genera as shown in Figure 4. In lab reared $C$. quinquefasciatus sample the OTUs were affiliated to following genera; Aeromonas, Asaia, Elizabethkingia, Enterobacter, Pseudomonas, Rahnella, Serratia and Wolbachia. Serratia marcescens was the most abundant species in this sample with a relative abundance of $64.29 \%$. Other species present in higher abundance were Rahnella uncultured bacterium 18.13\%, Serratia uncultured bacterium 5.08\%, Wolbachia Embioptera sp. $4.37 \%$ and Elizabethkingia meningoseptica $4.88 \%$ (Figure 4). However, in field collected Culex quinquefasciatus sample genera represented were, Wolbachia, Sphingomonas, Streptococcus, Serratia, Rhizobium, Rahnella, Pseudomonas, Methylobacterium, Ixodes, Helicobacter, Gamma proteobacterium, Enterobacter, Corynebacterium, Bartonella, Bacteroidetes, Bacillus, Asaia, Arcobacter, Akkermansia, Agrobacterium, and Aeromonas. The most abundant species in field collected C. quinquefasciatus sample were Arcobacter uncultured bacterium with relative abundance of $34.83 \%$, while Bartonella grahamii as4aup had $24.45 \%$ (Figure 4). Arcobacter uncultured bacterium, Bacteroidetes uncultured bacterium, B. grahamii as4up, Gamma Proteobacteria uncultured bacterium, Helicobacter $\mathrm{sp}$. B52Seymour and Ixodes scapularis were unique species in the field collected $C$. quinquefasciatus sample.

In lab reared $A$. gambiae sample, Asaia uncultured bacterium was the most abundant species with $39.30 \%$ relative abundance. Other taxa represented in the sample include Aeromonas sp. DMA1, Rahnella uncultured bacterium and Serratia marcescens each scoring a relative abundance of $10 \%$. The genera found in lab reared Anopheles gambiae sample include; Aeromonas, Serratia, Bacillus, Asaia, Chryseobacterium, Gluconacetobacter, Delftia, Pseudomonas, Rahnella, Thorsellia, Enterobacter and Stenotrophomonas. Thorsellia anophelis was unique to lab reared $A$. gambiae sample (Figure 5). The field collected $A$. gambiae sample was found to harbor a higher diversity of bacterial species. The most abundant species were Agrobacterium sp. $12.63 \%$ and Methylobacterium uncultured bacterium at $11.14 \%$ relative abundance. The most predominant genera found in field collected include; Serratia, Bacillus, Agrobacterium Stenotrophomonas, Gluconacetobacter, Methylobacterium. Rahnella (Figure 5). The unique species in field collected $A$. gambiae sample include 


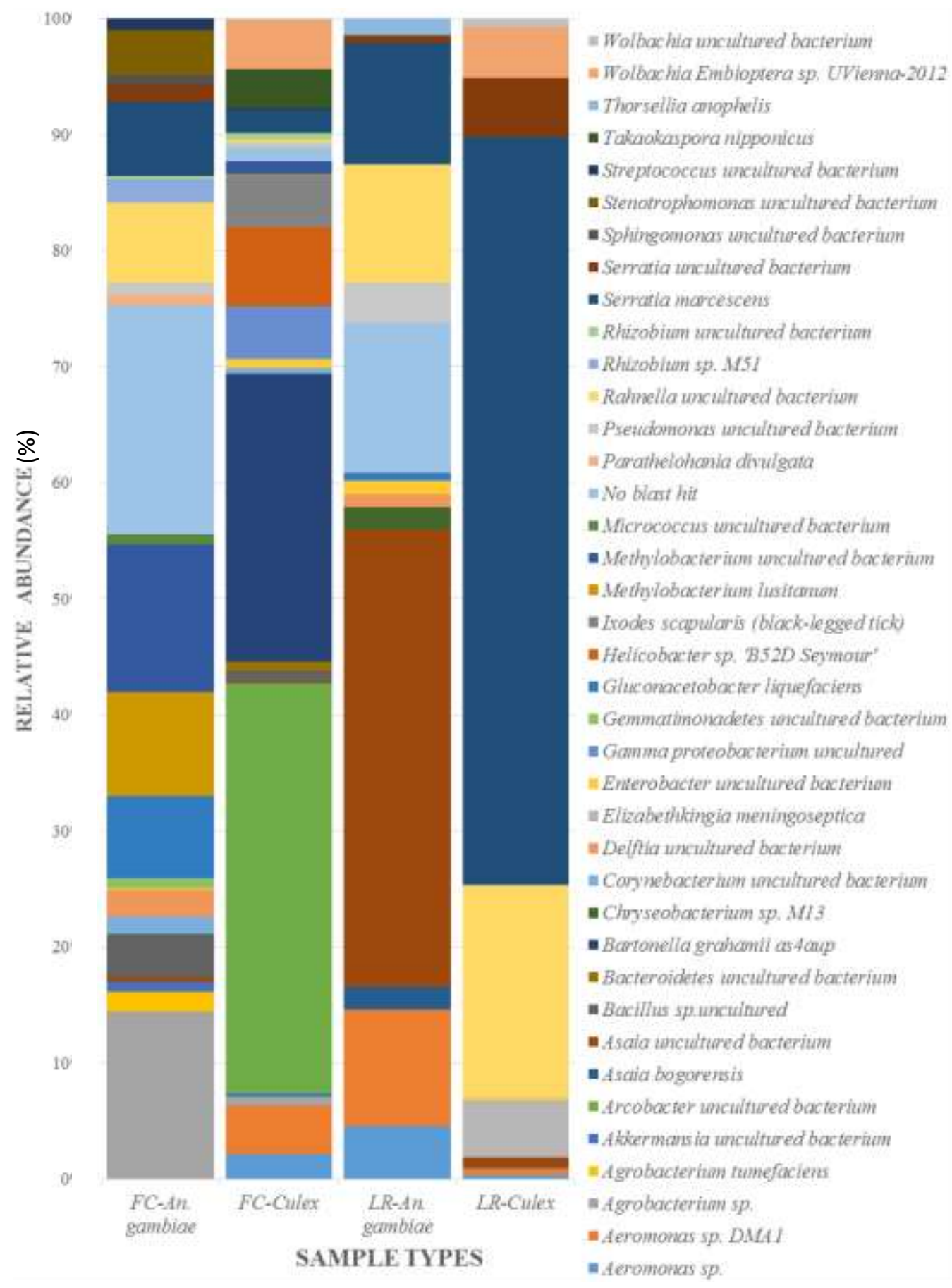

Figure 4. Relative abundance of the most predominant bacterial species from the field collected (FC) and lab reared (LR) samples.

Agrobacterium tumefaciens, Gemmatimonadetes uncultured bacterium, Micrococcus uncultured bacterium and Rhizobium sp. M51 (Figure 5).

Bacterial species which were recovered from all the four samples include, Serratia marcescens, Asaia uncultured bacterium, Enterobacter uncultured bacterium, Pseudomonas uncultured bacterium and Rahnella uncultured bacterium. Parathelohania divulgata and Takaokaspora nipponicus are fungal species recovered from the field collected $A$. gambiae and C.quinquefasciatus respectively (Figure 5 ). Detailed information on all the bacterial species recovered in this study is given in additional file 1 Table S1.

Hierarchical clustering, based on Bray-Curtis 


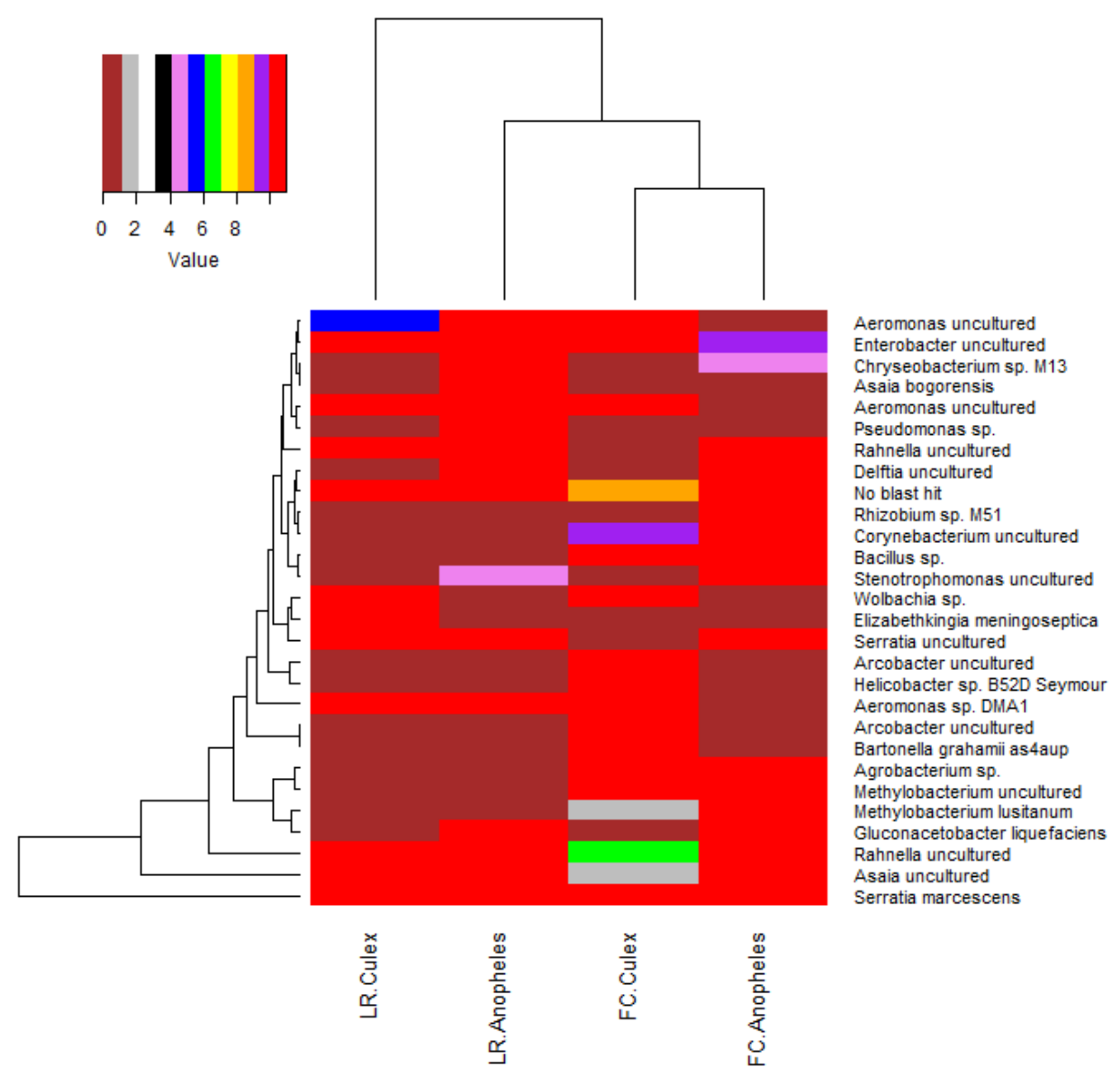

Figure 5. Hierarchical clustering of most abundant midgut bacterial species of the field collected (FC) and lab reared (LR) mosquitoes. Species level was chosen to be used in hierarchical clustering to assess the relationship between sample and taxa.

dissimilarity, showed two clusters (Figure 5). The dendogram shown on the top signify the relationship between the four samples. The bacteria composition of lab reared A.gambiae, field collected $A$. gambiae and field collected $C$. quinquefasciatus samples were clustered together. Within this cluster the field collected $A$. gambiae and field collected C. quinquefasciatus samples were more closely related to each other. The bacterial community recovered from the lab reared $C$. quinquefasciatus samples was observed to form a distinct cluster.

\section{DISCUSSION}

The microbes inhabiting mosquito midgut have drawn special attention in the recent past due to their interactions with both the mosquito host as well as disease causing parasites. The present study sought to investigate the composition and diversity of microbes in midguts of lab reared and field collected $A$. gambiae and C. quinquefasciatus mosquitoes. The field collected mosquitoes showed more midgut bacterial composition and diversity than the lab reared mosquitoes. A similar observation was reported by Rani et al. (2009) in their study involving the analysis of bacterial diversity in larvae and adult midgut microflora in lab reared and field collected Anopheles stephensi mosquito vectors. The higher bacterial diversity in field collected mosquitoes could probably be due to the fact that wild mosquitoes are exposed to the natural environment where they feed on various natural foods that could be the source of the diverse microbes, whereas the lab reared mosquitoes are fed on artificial diet of glucose and resins. Furthermore, adult female mosquitoes require a blood meal for their egg development and the blood acquired in the field could also be a source of various bacterial flora. On the other hand, the blood given to the adult female lab reared mosquitoes is from infection-free rabbits/mice (Rani et al., 2009). In the present study, the highest number of bacterial species was detected from field collected $A$. gambiae mosquitoes followed by field collected $C$. quinquefasciatus and lab reared $A$. gambiae. The least number of bacterial species were detected from lab 
reared C. quinquefasciatus.

Diversity indices analysis at OTU level from field collected and laboratory reared mosquitoes revealed a significant difference in microbial community composition. Field collected $A$. gambiae had the highest value of Inverse Simpson index and while the lowest was lab reared $C$. quinquefasciatus samples. The value of Inverse Simpson increases with diversity (Chandel et al., 2013). The Shannon index is another widely used index for comparing diversity between various habitats (Chandel et al., 2013). The Shannon index is a representation of both species abundance and evenness, when either of these two factors increase, the diversity index increases. Evenness was used for the estimating how well the species are evenly distributed among the samples. The lowest evenness was recorded from laboratory reared $C$. quinquefasciatus sample indicating that the bacterial species in this sample are less evenly distributed, that is, some species are more dominant than others. Comparative diversity was visualized using heatmap based on Bray-Curtis dissimilarities at species level. The microbial composition of the field collected samples at species level, were more similar compared to the laboratory reared. However, the laboratory reared samples the bacterial composition seemed to differ between $A$. gambiae and $C$. quinquefasciatus.

Members of the phylum Proteobacteria, were predominantly recovered from both the field collected and lab reared samples than those of phylum Firmicutes, Actinobacteria and Bacteriodetes. Proteobacteria were also shown to be dominant in a previous study conducted in Kenya on A. gambiae mosquitoes (Wang et al., 2011). Proteobacteria was the largest phylum represented by 43 species belonging to 26 genera. Some of the groups of bacteria recovered in the present study are similar to those recovered from previous culture dependent and culture-independent studies (Rani et al., 2009). Phylum Firmicutes consisted of ten species which were affiliated to nine genera. Actinobacteria represented fifteen species belonging to thirteen genera whereas Bacteriodetes consisted of five species belonging to five genera. Phylum Cyanobacteria, Gemmatimonadetes, Spirochaetae, Verrucomicrobia, Chloroflexi, Archeabacteria and Acidobacteria represented only a small portion of the mosquito midgut communities.

The dominant genera recovered in the present study belong to Serratia, Asaia, Arcobacter, Rahnella, Bartonella, Aeromonas, Agrobacterium, Methylobacterium and Wolbachia. The results of the study are consistent with earlier reports which have shown that that above genera are dominant (Pidiyar et al., 2004; Demaio et al., 1996; Favia et al., 2007; Dong et al., 2009). This suggests that at least a fraction of the mosquito midgut inhabitants could be common for different mosquito species inhabiting similar environments and may represent evolutionary conservation of association between bacteria and mosquito gut. Members of the genera Acinetobacter, Aeromonas, Bacillus, Enterobacter, Pseudomonas, Serratia, Asaia, Rahnella, and Stenotrophomonas have been frequently reported in mosquito gut in previous studies (Pidiyar et al., 2004; Boissière et al., 2012; Chandel et al., 2013). Sequences belonging to genera Asaia, Enterobacter, Pseudomonas, Rahnella and Serratia were recovered from all the samples and comprise a major part of microbiota of $A$. gambiae and $C$. quinquefasciatus mosquitoes in the present study.

Serratia marcescens appeared to be the core species $(23.6 \%)$ in the present study, as it was observed in both lab reared and field collected samples, suggesting that it possesses a competitive advantage over other bacterial species. S. marcescens is abundant in nature, and especially in the artificial foods given to the lab reared mosquitoes. Similar results were reported in five generations of lab reared $A$. gambiae (Dong et al., 2009).

Asaia uncultured bacterium species was recovered at $39.30 \%$ was more abundant in lab reared A.gambiae. Asaia has been associated with Anopheles species, in particular field collected Anopheles funestus, Anopheles Maculipennis, Anopheles gambiae and Anopheles coustani, and Anopheles stephensi in which Asaia bacteria was dominant and stably associated (Favia et al., 2007). The presence of Asaia species in Anopheles mosquito could be a target for malaria control it produces antiparasite molecules in mosquitoes that could be exploited in paratransgenic control of malaria (Damiani et al., 2010; Favia et al., 2007).

Elizabethkingia meningoseptica and Wolbachia sp. have repeatedly been detected in both lab reared and wild caught mosquitoes (Pumpuni et al., 1996) indicating their prevalent symbiotic association with mosquitoes. In the present study, Wolbacha was detected in both field collected and lab reared $C$. quinquefasciatus, previously it has been reported in several other mosquito vectors including, Aedes, Coquillettida and Masonia (Ricci et al., 2012).

Bacillus sp., Stenotrophomonas, Micrococcus Acinetobacter, and Rhizobium frequently isolated from soil and environmental samples were recovered at significantly greater numbers from the field collected mosquitoes. This suggests that the local soil and water environment plays an important role in colonization of the mosquito midgut at breeding sites or during nectar/blood feeding (Chandel et al., 2013). Parathelohania divulgata, Parathelohania obesa and Takaokaspora nipponicus fungal species were recovered from the field collected $A$. gambiae and $C$. quinquefasciatus but were absent in lab reared mosquitoes.

From the foregoing, the mosquito midgut has a rich diversity of symbiotic bacteria. The parasite mosquito relationship is believed to have been in existence for many years and it is likely that the acquired microflora permit the maintenance of pathogenic parasites in mosquitoes. The microbes could be benefiting the 
mosquitoes by protecting them against harmful bacteria and the mosquitoes could be benefiting the parasites by lowering the mosquito immunity against the parasites.

\section{Conclusion}

The results obtained present an analysis of the composition and bacterial diversity of lab reared and wild mosquitoes using Illumina sequencing technology. The bacterial flora of adult female $A$. gambiae and $C$. quinquefansciatus midgut is diverse and is dominated by bacterial species $S$. marcescens. In future, understanding the tripartite mosquito-microbes-parasite interaction will enable us gain more insight that may be useful in the development of novel approaches for the control of malaria and other mosquito borne diseases like filariasis, dengue, Zika and Chikungunya.

\section{CONFLICT OF INTERESTS}

\section{The authors have not declared any conflict of interests.}

\section{REFERENCES}

Agrawal V, Sashindran V (2006). Lymphatic Filariasis in India: Problems, Challenges and New Initiatives. Med. J. Armed Forces India 62:359-362.

Bando H, Okado K, Guelbeogo WM, Badolo A, Aonuma H, Nelson B, Fukumoto S, Xuan X, Sagnon NF, Kanuka H (2013). Intra-specific diversity of Serratia marcescens in Anopheles mosquito midgut defines Plasmodium transmission capacity. Sci. Rep. 3:1641.

Boissière $A$, Tchioffo $M T$, Bachar D, Abate L, Marie A, Nsango SE, Shahbazkia HR, Awono-Ambene PH, Levashina EA, Christen R, Morlais I (2012). Midgut Microbiota of the Malaria Mosquito Vector Anopheles gambiae and Interactions with Plasmodium falciparum Infection. PLoS Pathog. 8(5):e1002742.

Capone KA, Dowd SE, Stamatas GN, Nikolovski J (2011). Diversity of the Human Skin Microbiome Early in Life. J. Invest. Dermatol. 131:2026-2032.

Caporaso JG, Kuczynski J, Stombaugh J, Bittinger K, Bushman FD, Costello EK, Fierer N, Peña AG, Goodrich JK, Gordon JI, Huttley GA, Kelley ST, Knights D, Koenig JE, Ley RE, Lozupone CA, McDonald D, Muegge BD, Pirrung M, Reeder J, Sevinsky JR, Turnbaugh PJ, Walters WA, Widmann J, Yatsunenko T, Zaneveld J, Knight $\mathrm{R}$ (2010). QIIME allows analysis of high-throughput community sequencing data. Nat. Methods 7:335-336.

Centres for Disease Control and Prevention, CDC (2015). Malaria Malaria Worldwide - CDC-Activities in Kenya. Available at: http://www.cdc.gov/malaria/malaria_worldwide/cdc_activities/kenya.ht $\mathrm{ml}$.

Chandel K, Mendki MJ, Parikh RY, Kulkarni G, Tikar SN, Sukumaran D, Prakash S, Parashar BD, Shouche YS, Veer V (2013). Midgut Microbial Community of Culex quinquefasciatus Mosquito Populations from India. PLoS One 8(11):e80453.

Chao A, Gotelli NJ, Hsieh TC, Sander EL, Ma KH, Colwell RK, Ellison AM (2014). Rarefaction and extrapolation with Hill numbers: A framework for sampling and estimation in species diversity studies. Ecol. Monogr. 84:45-67.

Chavshin AR, Oshaghi MA, Vatandoost H, Pourmand MR, Raeisi A, Enayati AA, Mardani N, Ghoorchian S (2012). Identification of bacterial microflora in the midgut of the larvae and adult of wild caught Anopheles stephensi: a step toward finding suitable paratransgenesis candidates. Acta Trop. 121:129-134.

Damiani C, Ricci I, Crotti E, Rossi P, Rizzi A, Scuppa P, Capone A,
Ulissi U, Epis S, Genchi M, Sagnon N, Faye I, Kang A, Chouaia B, Whitehorn C, Moussa GW, Mandrioli M, Esposito F, Sacchi L, Bandi C, Daffonchio D, Favia G (2010). Mosquito-bacteria symbiosis: the case of Anopheles gambiae and Asaia. Microb. Ecol. 60:644-654.

Demaio J, Pumpuni CB, Kent M, Beier JC (1996). The midgut bacterial flora of wild Aedes triseriatus, Culex pipiens, and Psorophora columbiae mosquitoes. Am. J. Trop. Med. Hyg. 54:219-223.

Dong Y, Manfredini F, Dimopoulos G (2009). Implication of the Mosquito Midgut Microbiota in the Defense against Malaria Parasites. PLoS Pathog. 5:e1000423.

Dowd SE, Delton Hanson J, Rees E, Wolcott RD, Zischau AM, Sun Y, White J, Smith DM, Kennedy J, Jones CE (2011). Survey of fungi and yeast in polymicrobial infections in chronic wounds. J. Wound Care 20:40-47.

Eren AM, Zozaya M, Taylor CM, Dowd SE, Martin DH, Ferris MJ (2011). Exploring the Diversity of Gardnerella vaginalis in the Genitourinary Tract Microbiota of Monogamous Couples Through Subtle Nucleotide Variation. PLoS One 6(10):e26732.

Favia G, Ricci I, Damiani C, Raddadi N, Crotti E, Marzorati M, Rizzi A, Urso R, Brusetti L, Borin S, Mora D, Scuppa P, Pasqualini L, Clementi E, Genchi M, Corona S, Negri I, Grandi G, Alma A, Kramer L, Esposito F, Bandi C, Sacchi L, Daffonchio D (2007). Bacteria of the genus Asaia stably associate with Anopheles stephensi, an Asian malarial mosquito vector. Proc. Natl. Acad. Sci. U. S. A. 104:90479051.

Gillies MT, DE Meillon B (1968). The Anophelinae of Africa south of the Sahara (Ethiopian Zoogeographical Region). 343 p.

Njenga SM, Mwandawiro CS, Wamae CN, Mukoko DA, Omar AA, Shimada M, Bockarie MJ, Molyneux DH (2011). Sustained reduction in prevalence of lymphatic filariasis infection in spite of missed rounds of mass drug administration in an area under mosquito nets for malaria control. Parasit. Vectors 4(1):90.

Pidiyar VJ, Jangid K, Patole MS, Shouche YS (2004). Studies on Cultured and Uncultured Microbiota of Wild Culex quinquefasciatus Mosquito Midgut Based on 16s Ribosomal Rna Gene Analysis. Am. J. Trop. Med. Hyg. 70:597-603.

Pumpuni CB, Demaio J, Kent M, Davis JR, Beier JC (1996). Bacterial population dynamics in three anopheline species: the impact on Plasmodium sporogonic development. Am. J. Trop. Med. Hyg. 54:214-218.

Quast C, Pruesse E, Yilmaz P, Gerken J, Schweer T, Yarza P, Peplies J, Glöckner F O (2013). The SILVA ribosomal RNA gene database project: improved data processing and web-based tools. Nucleic Acids Res. 41:D590-D596.

R development Core Team (2012). R: A Language and Environment for Statistical Computing. $\mathrm{R}$ Foundation for Statistical Computing: Vienna, Austria.

Rani A, Sharma A, Rajagopal R, Adak T, Bhatnagar RK (2009). Bacterial diversity analysis of larvae and adult midgut microflora using culture-dependent and culture-independent methods in labreared and field-collected Anopheles stephensi-an Asian malarial vector. BMC Microbiol. 9(1):96.

Ricci I, Damiani C, Capone A, DeFreece C, Rossi P, Favia G (2012). Mosquito/microbiota interactions: from complex relationships to biotechnological perspectives. Curr. Opin. Microbiol. 15:278-284.

Riehle MA, Jacobs-Lorena M (2005). Using bacteria to express and display anti-parasite molecules in mosquitoes: current and future strategies. Insect Biochem. Mol. Biol. 35:699-707.

Wang Y, Gilbreath TM, Kukutla P, Yan G, Xu J (2011). Dynamic Gut Microbiome across Life History of the Malaria Mosquito Anopheles gambiae in Kenya. PLoS One 6:e24767.

Whitten MMA, Shiao SH, Levashina EA (2006). Mosquito midguts and malaria: cell biology, compartmentalization and immunology. Parasite Immunol. 28:121-130.

WHO- World Health Organisation (2015). World malaria report. http://www.who.int/malaria/publications/world-malaria-report2015/report/en/.

Yu K, Zhang T (2012). Metagenomic and Metatranscriptomic Analysis of Microbial Community Structure and Gene Expression of Activated Sludge. PLoS One 7(5):e38183. 


\section{Additional file 1}

Table S1. Midgut bacterial composition at species level and their abundance.

\begin{tabular}{|c|c|c|c|c|c|}
\hline Species level & FC.Anopheles & FC.Culex & LR.Anopheles & LR.Culex & Total \\
\hline \multicolumn{6}{|l|}{ Phylum Archeabacteria } \\
\hline Archaea, Euryarchaeota, Methanobacteria, Methanobacteriales, Methanobacteriaceae, Methanobrevibacter, uncultured archaeon & 24 & 0 & 0 & 0 & \\
\hline \multicolumn{6}{|l|}{ Phylum Acidobacteria } \\
\hline Bacteria, Acidobacteria, Acidobacteria, Subgroup 4, Unknown Family, Blastocatella, uncultured Acidobacteria bacterium & 10 & 0 & 0 & 0 & \\
\hline Bacteria, Acidobacteria, Acidobacteria, Subgroup 6, uncultured Acidobacteria bacterium & 32 & 0 & 0 & 0 & \\
\hline \multicolumn{6}{|l|}{ Phylum Actinobacteria } \\
\hline Bacteria, Actinobacteria, Actinobacteria, Corynebacteriales, Corynebacteriaceae, Corynebacterium uncultured bacterium & 72 & 10 & 0 & 0 & \\
\hline Bacteria, Actinobacteria, Actinobacteria, Corynebacteriales, Corynebacteriaceae, Corynebacterium, uncultured Corynebacterium sp. & 6 & 6 & 0 & 0 & \\
\hline Bacteria, Actinobacteria, Actinobacteria, Corynebacteriales, Corynebacteriaceae, Corynebacterium unidentified marine bacterioplankton & 10 & 3 & 0 & 0 & \\
\hline Bacteria, Actinobacteria, Actinobacteria, Corynebacteriales, Corynebacteriaceae, uncultured, uncultured bacterium & 31 & 1 & 0 & 0 & \\
\hline Bacteria, Actinobacteria, Actinobacteria, Corynebacteriales, Dietziaceae, Dietzia uncultured bacterium & 8 & 0 & 0 & 0 & \\
\hline Bacteria, Actinobacteria, Actinobacteria, Frankiales, Geodermatophilaceae, Blastococcus uncultured bacterium & 7 & 0 & 0 & 0 & \\
\hline Bacteria, Actinobacteria, Actinobacteria, Micrococcales, Cellulomonadaceae, Actinotalea uncultured bacterium & 17 & 0 & 0 & 0 & \\
\hline Bacteria, Actinobacteria, Actinobacteria, Micrococcales, Intrasporangiaceae, Terrabacter uncultured bacterium & 38 & 1 & 0 & 0 & \\
\hline Bacteria, Actinobacteria, Actinobacteria, Micrococcales, Microbacteriaceae, Curtobacterium uncultured bacterium & 24 & 0 & 0 & 0 & \\
\hline Bacteria, Actinobacteria, Actinobacteria, Micrococcales, Micrococcaceae, Arthrobacter, Arthrobacter sp. TSBY-23 & 7 & 0 & 0 & 0 & \\
\hline Bacteria, Actinobacteria, Actinobacteria, Micrococcales, Micrococcaceae, Enteractinococcus, Yaniella sp. YUAB-SO-24 & 7 & 1 & 0 & 0 & \\
\hline Bacteria, Actinobacteria, Actinobacteria, Micrococcales, Micrococcaceae, Kocuria, Kocuria sp. oral clone AW006 & 11 & 2 & 0 & 0 & \\
\hline Bacteria, Actinobacteria, Actinobacteria, Micrococcales, Micrococcaceae, Micrococcus uncultured bacterium & 43 & 0 & 0 & 0 & \\
\hline Bacteria, Actinobacteria, Actinobacteria, Propionibacteriales, Nocardioidaceae, Nocardioides uncultured bacterium & 4 & 2 & 0 & 0 & \\
\hline Bacteria, Actinobacteria, Actinobacteria, Streptomycetales, Streptomycetaceae, Streptomyces, Streptomyces ferralitis & 12 & 0 & 0 & 0 & \\
\hline Bacteria, Actinobacteria, Thermoleophilia, Gaiellales, uncultured, uncultured bacterium & 12 & 0 & 0 & 0 & \\
\hline \multicolumn{6}{|l|}{ Phylum Bacteroidetes } \\
\hline Bacteria, Bacteroidetes, Bacteroidia, Bacteroidales, Rikenellaceae, RC9 gut group, uncultured bacterium & 6 & 0 & 0 & 0 & \\
\hline Bacteria, Bacteroidetes, Flavobacteriia, Flavobacteriales, Cryomorphaceae, Fluviicola uncultured bacterium & 14 & 0 & 0 & 0 & \\
\hline Bacteria, Bacteroidetes, Flavobacteriia, Flavobacteriales, Flavobacteriaceae, Chryseobacterium, Chryseobacterium sp. M13 & 5 & 0 & 113 & 0 & \\
\hline Bacteria, Bacteroidetes, Flavobacteriia, Flavobacteriales, Flavobacteriaceae, Elizabethkingia, Elizabethkingia meningoseptica & 1 & 0 & 0 & 291 & \\
\hline Bacteria, Bacteroidetes, Flavobacteriia, Flavobacteriales, Flavobacteriaceae, uncultured, uncultured Bacteroidetes bacterium & 0 & 21 & 0 & 0 & \\
\hline \multicolumn{6}{|l|}{ Phylum Chloroflexi } \\
\hline Bacteria, Chloroflexi, Thermomicrobia, JG30-KF-CM45, uncultured soil bacterium & 31 & 0 & 0 & 0 & \\
\hline Bacteria, Chloroflexi, Thermomicrobia, Sphaerobacterales, Sphaerobacteraceae, Nitrolancea, uncultured Chloroflexi bacterium & 19 & 0 & 0 & 0 & \\
\hline \multicolumn{6}{|l|}{ Phylum Cyanobacteria } \\
\hline Bacteria, Cyanobacteria, Chloroplast uncultured bacterium & 6 & 0 & 0 & 0 & \\
\hline \multicolumn{6}{|l|}{ Phylum Firmicutes } \\
\hline Bacteria, Firmicutes, Bacilli, Bacillales, Bacillaceae, Anoxybacillus uncultured bacterium & 0 & 10 & 1 & 0 & \\
\hline Bacteria, Firmicutes, Bacilli, Bacillales, Bacillaceae, Bacillus, uncultured Bacillus sp. & 183 & 31 & 1 & 0 & \\
\hline
\end{tabular}


Table S1. Contd.

Bacteria, Firmicutes, Bacilli, Bacillales, Bacillaceae, Bacillus uncultured bacterium

Bacteria, Firmicutes, Bacilli, Bacillales, Planococcaceae, Lysinibacillus uncultured bacterium

Bacteria, Firmicutes, Bacilli, Bacillales, Staphylococcaceae, Salinicoccus uncultured bacterium

Bacteria, Firmicutes, Bacilli, Lactobacillales, Carnobacteriaceae, Atopostipes uncultured bacterium

Bacteria, Firmicutes, Bacilli, Lactobacillales, Carnobacteriaceae, uncultured, uncultured bacterium

Bacteria, Firmicutes, Bacilli, Lactobacillales, Streptococcaceae, Streptococcus uncultured bacterium

Bacteria, Firmicutes, Clostridia, Clostridiales, Peptostreptococcaceae, Incertae Sedis uncultured bacterium

Bacteria, Firmicutes, Clostridia, Clostridiales, Ruminococcaceae, uncultured, uncultured bacterium

\section{Phylum Gemmatimonadetes}

Bacteria, Gemmatimonadetes, Gemmatimonadetes, AT425-EubC11 terrestrial group, uncultured bacterium

\section{Phylum Proteobacteria}

Bacteria, Proteobacteria, Alphaproteobacteria, Caulobacterales, Caulobacteraceae, Brevundimonas uncultured bacterium

Bacteria, Proteobacteria, Alphaproteobacteria, Rhizobiales, Bartonellaceae, Bartonella, Bartonella grahamii as4aup

Bacteria, Proteobacteria, Alphaproteobacteria, Rhizobiales, Bradyrhizobiaceae, Bosea, uncultured Bosea sp.

Bacteria, Proteobacteria, Alphaproteobacteria, Rhizobiales, Methylobacteriaceae, Methylobacterium, Methylobacterium lusitanum

Bacteria, Proteobacteria, Alphaproteobacteria, Rhizobiales, Methylobacteriaceae, Methylobacterium uncultured bacterium

Bacteria, Proteobacteria, Alphaproteobacteria, Rhizobiales, Rhizobiaceae, Rhizobium, Agrobacterium tumefaciens

Bacteria, Proteobacteria, Alphaproteobacteria, Rhizobiales, Rhizobiaceae, Rhizobium, Rhizobium sp. JC140

Bacteria, Proteobacteria, Alphaproteobacteria, Rhizobiales, Rhizobiaceae, Rhizobium, Rhizobium sp. M5

Bacteria, Proteobacteria, Alphaproteobacteria, Rhizobiales, Rhizobiaceae, Rhizobium, uncultured Agrobacterium sp.

Bacteria, Proteobacteria, Alphaproteobacteria, Rhizobiales, Rhizobiaceae, Rhizobium uncultured bacterium

Bacteria, Proteobacteria, Alphaproteobacteria, Rhizobiales, Rhizobiaceae, Rhizobium, uncultured Paracoccus sp.

Bacteria, Proteobacteria, Alphaproteobacteria, Rhodobacterales, Rhodobacteraceae, Paracoccus uncultured bacterium

Bacteria, Proteobacteria, Alphaproteobacteria, Rhodospirillales, Acetobacteraceae, Acetobacter, uncultured Acetobacter sp.

Bacteria, Proteobacteria, Alphaproteobacteria, Rhodospirillales, Acetobacteraceae, Asaia, Asaia bogorensis

Bacteria, Proteobacteria, Alphaproteobacteria, Rhodospirillales, Acetobacteraceae, Asaia uncultured bacterium

Bacteria, Proteobacteria, Alphaproteobacteria, Rhodospirillales, Acetobacteraceae, Asaia uncultured bacterium

Bacteria, Proteobacteria, Alphaproteobacteria, Rhodospirillales, Acetobacteraceae, Asaia uncultured bacterium

Bacteria, Proteobacteria, Alphaproteobacteria, Rhodospirillales, Acetobacteraceae, Gluconacetobacter, Gluconacetobacter liquefaciens

Bacteria, Proteobacteria, Alphaproteobacteria, Rickettsiales, Anaplasmataceae, Wolbachia Embioptera sp. UVienna-2012

Bacteria, Proteobacteria, Alphaproteobacteria, Rickettsiales, Anaplasmataceae, Wolbachia uncultured bacterium

Bacteria, Proteobacteria, Alphaproteobacteria, Rickettsiales, mitochondria, Triticum aestivum

Bacteria, Proteobacteria, Alphaproteobacteria, Rickettsiales, Rickettsiaceae, Rickettsia, uncultured Rickettsia sp.

Bacteria, Proteobacteria, Alphaproteobacteria, Sphingomonadales, Sphingomonadaceae, Sphingomonas uncultured bacterium

Bacteria, Proteobacteria, Alphaproteobacteria, Sphingomonadales, Sphingomonadaceae, Sphingomonas, uncultured Firmicutes bacterium

Bacteria, Proteobacteria, Betaproteobacteria, Burkholderiales, Comamonadaceae, Delftia uncultured bacterium

Bacteria, Proteobacteria, Betaproteobacteria, Burkholderiales, Comamonadaceae, Delftia, uncultured Delftia sp.

Bacteria, Proteobacteria, Betaproteobacteria, Burkholderiales, Oxalobacteraceae, Oxalicibacterium, Oxalicibacterium flavum

Bacteria, Proteobacteria, Betaproteobacteria, Neisseriales, Neisseriaceae, uncultured, uncultured bacterium

\begin{tabular}{|c|c|c|c|}
\hline 5 & 1 & 0 & 0 \\
\hline 2 & 5 & 0 & 0 \\
\hline 39 & 0 & 0 & 0 \\
\hline 6 & 1 & 0 & 0 \\
\hline 6 & 0 & 0 & 0 \\
\hline 3 & 4 & 0 & 0 \\
\hline 6 & 4 & 1 & 0 \\
\hline & 0 & 0 & 0 \\
\hline 2 & 0 & 0 & 0 \\
\hline 2 & 0 & 0 & 0 \\
\hline 0 & 737 & 0 & 0 \\
\hline 37 & 0 & 0 & 0 \\
\hline 68 & 2 & 0 & 0 \\
\hline 65 & 32 & 0 & 0 \\
\hline 37 & 0 & 0 & 0 \\
\hline 3 & 0 & 0 & 0 \\
\hline 06 & 0 & 0 & 0 \\
\hline 54 & 21 & 0 & 0 \\
\hline 0 & 18 & 0 & 0 \\
\hline 9 & 1 & 0 & 0 \\
\hline 9 & 0 & 0 & 0 \\
\hline 0 & 0 & 8 & 0 \\
\hline 0 & 0 & 116 & 1 \\
\hline 6 & 2 & 2223 & 55 \\
\hline 0 & 0 & 47 & 0 \\
\hline 5 & 0 & 39 & 0 \\
\hline 70 & 0 & 37 & 0 \\
\hline 0 & 125 & 0 & 261 \\
\hline 0 & 5 & 0 & 43 \\
\hline 7 & 0 & 0 & 0 \\
\hline 9 & 0 & 0 & 0 \\
\hline 1 & 2 & 0 & 0 \\
\hline 24 & 0 & 0 & 0 \\
\hline 4 & 0 & 59 & 0 \\
\hline 1 & 0 & 7 & 0 \\
\hline 30 & 0 & 0 & 0 \\
\hline 7 & 1 & 0 & 0 \\
\hline
\end{tabular}


Table S1. Contd.

Bacteria, Proteobacteria, Epsilonproteobacteria, Campylobacterales, Campylobacteraceae, Arcobacter uncultured bacterium Bacteria, Proteobacteria, Epsilonproteobacteria, Campylobacterales, Helicobacteraceae, Helicobacter, Helicobacter sp. 'B52D Seymour' Bacteria, Proteobacteria, Gammaproteobacteria, Aeromonadales, Aeromonadaceae, Aeromonas, Aeromonas sp. DMA1

Bacteria, Proteobacteria, Gammaproteobacteria, Aeromonadales, Aeromonadaceae, Aeromonas, uncultured Aeromonas sp. Bacteria, Proteobacteria, Gammaproteobacteria, Aeromonadales, Aeromonadaceae, Aeromonas uncultured bacterium Bacteria, Proteobacteria, Gammaproteobacteria, Enterobacteriales, Enterobacteriaceae, Enterobacter uncultured bacterium Bacteria, Proteobacteria, Gammaproteobacteria, Enterobacteriales, Enterobacteriaceae, Escherichia-Shigella, Serratia marcescens Bacteria, Proteobacteria, Gammaproteobacteria, Enterobacteriales, Enterobacteriaceae, Rahnella uncultured bacterium Bacteria, Proteobacteria, Gammaproteobacteria, Enterobacteriales, Enterobacteriaceae, Rahnella uncultured bacterium Bacteria, Proteobacteria, Gammaproteobacteria, Enterobacteriales, Enterobacteriaceae, Rahnella uncultured bacterium Bacteria, Proteobacteria, Gammaproteobacteria, Enterobacteriales, Enterobacteriaceae, Serratia, Serratia marcescens Bacteria, Proteobacteria, Gammaproteobacteria, Enterobacteriales, Enterobacteriaceae, Serratia, Serratia marcescens Bacteria, Proteobacteria, Gammaproteobacteria, Enterobacteriales, Enterobacteriaceae, Serratia, Serratia marcescens Bacteria, Proteobacteria, Gammaproteobacteria, Enterobacteriales, Enterobacteriaceae, Serratia, Serratia marcescens Bacteria, Proteobacteria, Gammaproteobacteria, Enterobacteriales, Enterobacteriaceae, Serratia, Serratia marcescens Bacteria, Proteobacteria, Gammaproteobacteria, Enterobacteriales, Enterobacteriaceae, Serratia, Serratia sp. DR.Y5 Bacteria, Proteobacteria, Gammaproteobacteria, Enterobacteriales, Enterobacteriaceae, Serratia uncultured bacterium Bacteria, Proteobacteria, Gammaproteobacteria, Enterobacteriales, Enterobacteriaceae, Serratia uncultured bacterium Bacteria, Proteobacteria, Gammaproteobacteria, Enterobacteriales, Enterobacteriaceae, Serratia uncultured bacterium Bacteria, Proteobacteria, Gammaproteobacteria, Enterobacteriales, Enterobacteriaceae, Serratia uncultured bacterium Bacteria, Proteobacteria, Gammaproteobacteria, Enterobacteriales, Enterobacteriaceae, Serratia uncultured bacterium Bacteria, Proteobacteria, Gammaproteobacteria, Enterobacteriales, Enterobacteriaceae, Thorsellia, Thorsellia anophelis Bacteria, Proteobacteria, Gammaproteobacteria, Orbales, Orbaceae, Gilliamella, uncultured gamma proteobacterium Bacteria, Proteobacteria, Gammaproteobacteria, Orbales, Orbaceae, Gilliamella, uncultured gamma proteobacterium Bacteria, Proteobacteria, Gammaproteobacteria, Pseudomonadales, Moraxellaceae, Acinetobacter, Acinetobacter sp. B7_2TCO2 Bacteria, Proteobacteria, Gammaproteobacteria, Pseudomonadales, Moraxellaceae, Acinetobacter uncultured bacterium Bacteria, Proteobacteria, Gammaproteobacteria, Pseudomonadales, Moraxellaceae, Acinetobacter uncultured bacterium Bacteria, Proteobacteria, Gammaproteobacteria, Pseudomonadales, Moraxellaceae, Enhydrobacter uncultured proteobacterium Bacteria, Proteobacteria, Gammaproteobacteria, Pseudomonadales, Moraxellaceae, Psychrobacter uncultured bacterium Bacteria, Proteobacteria, Gammaproteobacteria, Pseudomonadales, Pseudomonadaceae, Pseudomonas uncultured bacterium Bacteria, Proteobacteria, Gammaproteobacteria, Pseudomonadales, Pseudomonadaceae, Pseudomonas uncultured bacterium Bacteria, Proteobacteria, Gammaproteobacteria, Pseudomonadales, Pseudomonadaceae, Pseudomonas uncultured Pseudomonas sp. Bacteria, Proteobacteria, Gammaproteobacteria, Xanthomonadales, Xanthomonadaceae, Stenotrophomonas uncultured bacterium Bacteria, Proteobacteria, Gammaproteobacteria, Xanthomonadales, Xanthomonadaceae, Stenotrophomonas, uncultured bacterium Bacteria, Proteobacteria, Gammaproteobacteria, Xanthomonadales, Xanthomonadales Incertae Sedis, Steroidobacter uncultured bacterium Phylum Spirochaetae

Bacteria,Spirochaetae,Spirochaetes,Spirochaetales,Spirochaetaceae,uncultured,Spironema culicis

Phylum Verrucomicrobia

Bacteria,Verrucomicrobia,Verrucomicrobiae,Verrucomicrobiales,Verrucomicrobiaceae,Akkermansiauncultured bacterium

\begin{tabular}{|c|c|c|c|}
\hline & 1050 & 0 & 0 \\
\hline & 204 & 0 & 0 \\
\hline & 125 & 588 & 37 \\
\hline & 0 & 12 & 2 \\
\hline & 64 & 254 & 18 \\
\hline & 23 & 71 & 13 \\
\hline & 0 & 1 & 4 \\
\hline 8 & 7 & 399 & 1050 \\
\hline 0 & 0 & 210 & 31 \\
\hline 2 & 0 & 0 & 0 \\
\hline 88 & 48 & 365 & 2146 \\
\hline 5 & 14 & 232 & 1236 \\
\hline & 0 & 15 & 98 \\
\hline 1 & 3 & 69 & 399 \\
\hline & 0 & 7 & 23 \\
\hline & 0 & 1 & 13 \\
\hline & 0 & 4 & 18 \\
\hline & 0 & 0 & 1 \\
\hline 2 & 0 & 12 & 59 \\
\hline & 0 & 11 & 91 \\
\hline 9 & 0 & 10 & 53 \\
\hline & 0 & 81 & 0 \\
\hline & 69 & 0 & 0 \\
\hline & 65 & 0 & 0 \\
\hline & 0 & 0 & 0 \\
\hline & 3 & 0 & 0 \\
\hline & 1 & 1 & 0 \\
\hline 3 & 4 & 0 & 0 \\
\hline 1 & 0 & 0 & 0 \\
\hline 1 & 14 & 26 & 6 \\
\hline 8 & 0 & 0 & 0 \\
\hline & 0 & 177 & 0 \\
\hline 6 & 0 & 5 & 0 \\
\hline 5 & 0 & 0 & 0 \\
\hline 6 & 0 & 0 & 0 \\
\hline & 6 & 0 & 0 \\
\hline 7 & 0 & 0 & 0 \\
\hline
\end{tabular}


Table S1. Contd.

Bacteria,Verrucomicrobia,Verrucomicrobiae,Verrucomicrobiales,Verrucomicrobiaceae,Akkermansiauncultured bacterium

Phylum Eukaryota

Eukaryota, Opisthokonta, Nucletmycea, Fungi, Microsporidia, Incertae Sedis, Amblyosporidae, Parathelohania, Parathelohania divulgata

Eukaryota, Opisthokonta, Nucletmycea, Fungi, Microsporidia, Incertae Sedis, Amblyosporidae, Parathelohania Parathelohania obesa

Eukaryota, Opisthokonta, Nucletmycea, Fungi, Microsporidia, Incertae Sedis, Amblyosporidae, Takaokaspora Takaokaspora nipponicus

No blast hit
0

51

33

0

1030

6214
7

0

0

100

34
0

0

Total abundance

2890
0

$0 \quad 0$

$757 \quad 0$

$5960 \quad 5949$

